\title{
3GPP Channel Model Emulation with Analysis of MIMO-LTE Performances in Reverberation Chamber
}

\author{
Nabil Arsalane, Moctar Mouhamadou, Cyril Decroze, David Carsenat, \\ Miguel Angel Garcia-Fernandez, and Thierry Monedière
}

XLim Laboratory, OSA Department, UMR CNRS 6172, University of Limoges, 123 Avenue Albert Thomas,
87060 Limoges Cedex, France

Correspondence should be addressed to Nabil Arsalane, nabil.arsalane@xlim.fr

Received 2 December 2011; Accepted 24 January 2012

Academic Editor: David A. Sanchez-Hernandez

Copyright (๑) 2012 Nabil Arsalane et al. This is an open access article distributed under the Creative Commons Attribution License, which permits unrestricted use, distribution, and reproduction in any medium, provided the original work is properly cited.

\begin{abstract}
Emulation methodology of multiple clusters channels for evaluating wireless communication devices over-the-air (OTA) performance is investigated. This methodology has been used along with the implementation of the SIMO LTE standard. It consists of evaluating effective diversity gain (EDG) level of SIMO LTE-OFDM system for different channel models according to the received power by establishing an active link between the transmitter and the receiver. The measurement process is set up in a Reverberation Chamber (RC). The obtained results are compared to the reference case of single input-single output (SISO) in order to evaluate the real improvement attained by the implemented system.
\end{abstract}

\section{Introduction}

In recent research works, reverberation chamber (RC) is considered as a useful tool to emulate rich multipath environments $[1,2]$. In this contribution, this tool is employed for emulating multiclusters channel models. Indeed, RC represents a useful candidate for performance evaluation of wireless communication systems, and they are being considered as a standard for multiple-input multiple-output (MIMO) over-the-air (OTA) measurements in 3GPP and CTIA standardization committees.

Active measurement methods are often based on the use of a channel emulator associated with a real-time transmission system, to test the operational terminals. In this paper, the aim is to suggest an experimental platform using a small size reverberation chamber (reverberant cell) to study the feasibility of emulating multipath channel while maintaining a Rayleigh fading (in order to be able to compare different receivers in reference environments with the same distribution). On this platform, a multicluster emulation method which complies with channels defined by $3 \mathrm{GPP}$ models is implemented, using only one vector signal generator. This emulation must be accompanied by a strict control of delay spread, to generate realistic channels [2-4].
This methodology, along with the presented model, emulates a Spatial-Channel-Model-Extended (SCME) for MIMO OTA active measurements. The delay spread control can be achieved through modifying the RC quality factor by loading it with absorbing materials.

The presented approach aims to develop a flexible OTA methodology for quantification and implementation of digital multiantenna transmission systems inside a small size reverberant cell. On this test bed, the measurements are not carried out in real time and are not dedicated to performance evaluation in terms of throughput. However, it allows (through the use of an RF digitizer and baseband processing in MATLAB) to study in detail the influence of several transmission chain parameters, as antenna aspects (in MIMO context: coupling, correlation coefficient $\rho$ ), and test of signal shaping and reception algorithms (synchronization, equalization, MIMO coding).

In this paper, this method is applied to test the 3GPP LTE standard, by implementing an LTE-OFDM frame and using diversity at the receiver side. The frame is generated based on the 3GPP standard $[5,6]$, which specifies a downlink (DL) transmission system using an orthogonal frequency division multiplexing access (OFDMA) [7, 8]. 


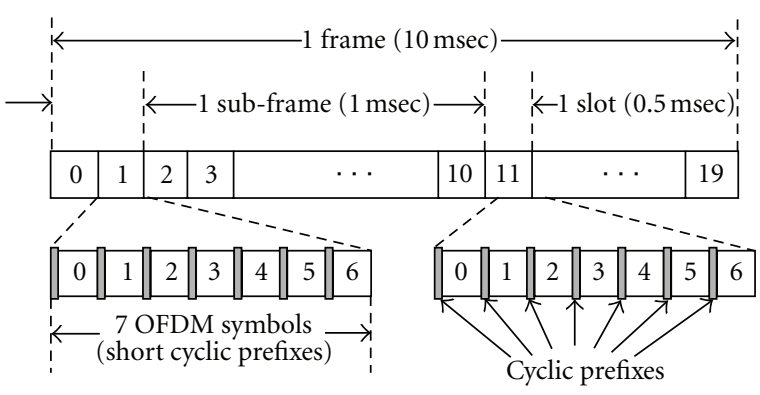

FIgURE 1: LTE Frame [5].
TABle 1: Parameters of the urban microcell and urban macrocell scenarios.

\begin{tabular}{|c|c|c|c|c|}
\hline \multirow{2}{*}{ Scenario } & \multicolumn{2}{|c|}{ Urban Macro } & \multicolumn{2}{|c|}{ Urban micro } \\
\hline & $\begin{array}{c}\text { Relative } \\
\text { power }(\mathrm{dB})\end{array}$ & Delay $(\mu \mathrm{s})$ & $\begin{array}{c}\text { Relative } \\
\text { power }(\mathrm{dB})\end{array}$ & Delay $(\mu \mathrm{s})$ \\
\hline \multirow{6}{*}{ Taps Model } & 0 & 0 & 0 & 0 \\
\hline & -2.22 & 0.36 & -1.27 & 0.28 \\
\hline & -1.72 & 0.25 & -2.72 & 0.20 \\
\hline & -5.72 & 1.04 & -4.30 & 0.66 \\
\hline & -9.05 & 2.7 & -6.01 & 0.81 \\
\hline & -12.50 & 4.59 & -8.43 & 0.92 \\
\hline
\end{tabular}

baseband digital IQ symbols [16]. Data processing is done with MATLAB.

At the transmitter side, a frame based on LTE specifications is generated. The duplex mode used is TDD, and a bandwidth of $5 \mathrm{MHz}$ has been chosen, with 64QAM modulation scheme, over a carrier frequency of $2.35 \mathrm{GHz}$.

\section{Channel Emulation}

The characteristics of the LTE-OFDM frame and measurement system to be used has been explained previously. This section will focus on establishing a method for the emulation of 3 GPP channel models with a specific delay spread, which requires a control of the delay spread inside the RC.

3.1. 3GPP Channel Model. The 3GPP urban microcell and urban macrocell channel models in $[5,6]$ are defined to be used for multiantenna OTA comparison measurements. The taps delay in urban micro-cell and urban macro-cell channel models are depicted in Figure 3.

It can be seen that the urban macrocell channel presents a high delay spread compared to the urban microcell.

The taps delay and the power magnitude are listed in details in Table 1.

Some special consideration should be taken into account when implementing channel models in an RC. Indeed these models introduce intracluster delay spread. Then, for each tap an RMS delay spread $\left(\tau_{\text {rms }}\right)$ of $90 \pm 5$ ns has to be considered.

\subsection{Channel Model Emulation}

3.2.1. Controlling the RMS Delay Spread. In order to obtain the desired $\tau_{\text {rms }}$, we first face up to the question related to chamber loading.

The RC used in this work is the SMART 1000 Mini Reverb-cell [17], which is a rectangular metallic enclosure with dimensions of $110 \mathrm{~cm} \times 70 \mathrm{~cm} \times 60 \mathrm{~cm}$, as shown in Figure 4 . The stirring operation is performed by vertical and horizontal stirrers and illuminated by two horn antennas connected to the transmitter.

The delay spread $\tau_{\text {rms }}$ can be modified by loading the chamber with an appropriate amount of absorbing material $[3,4]$. 


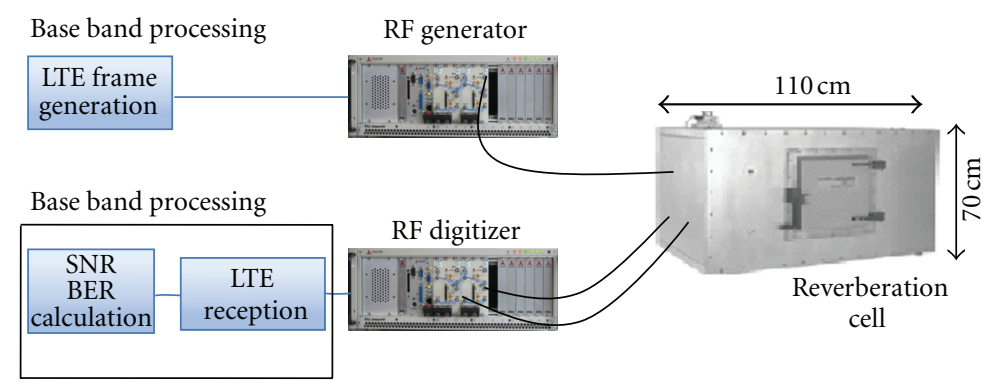

Figure 2: Measurement test bed.

TABLE 2: Diversity system under test characteristics.

\begin{tabular}{lcccc}
\hline Frequency $(\mathrm{GHz})$ & Return losses S11 $(\mathrm{dB})$ & Coupling coefficient S21 (dB) & $\begin{array}{c}\text { Correlation coefficient } \\
(\text { in isotropic environment) }\end{array}$ & Total efficiency $(\%)$ \\
\hline 2.35 & -12 & -17 & $<0.05$ & 92.7 \\
\hline
\end{tabular}

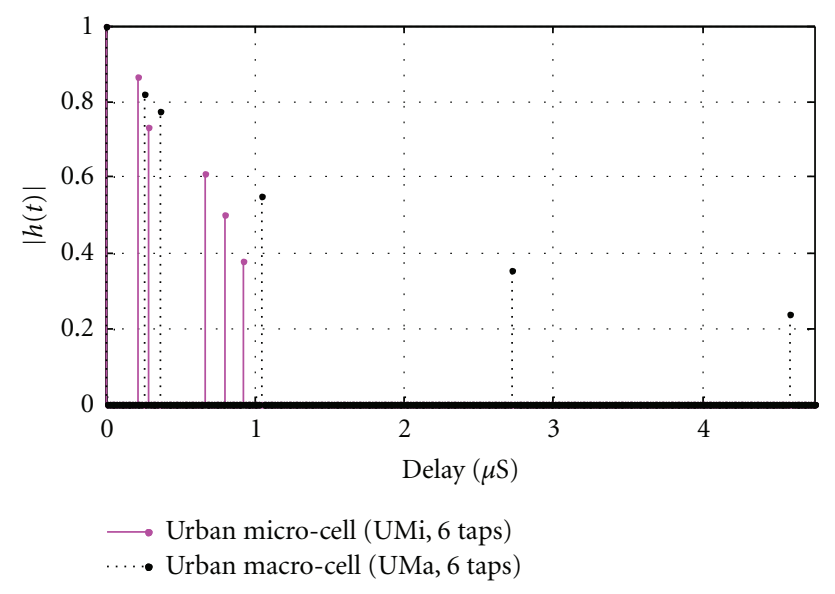

FIGURE 3: Urban micro- and macrocell channel model taps.

The measurements shown in Figure 5 illustrate the dependence of the delay spread on absorbing material located inside the RC.

For the unloaded chamber case, the measured RMS delay spread is equal to $256.4 \mathrm{~ns}$. When the chamber is loaded with a piece of material, which is solid, pyramidal shaped, and carbon loaded (urethane foam absorber [18]) with dimensions of $16 \mathrm{~cm} \times 9 \mathrm{~cm} \times 4 \mathrm{~cm}$, placed in a corner of the RC, the RMS delay spread is around $93 \mathrm{~ns}$. The use of absorbing materials decreases considerably the value of the delay spread and allows achieving the desired result which should be within $90 \pm 5 \mathrm{~ns}$.

In order to check the fading distribution, a power measurement is made at the central frequency to plot the normalized CDF. Figure 6 shows that the fading is still Rayleigh even after the addition of losses. The difference concerning the measured mean powers before and after adding absorbing materials is around $3 \mathrm{~dB}$.

In this part, the control of the $\tau_{\text {rms }}$ has been achieved by the use of absorbing material, and maintaining a Rayleigh fading distribution. The goal now is to emulate a multi-cluster channel with the same delay spread for each cluster.

3.2.2. Clusters Emulation. The method to emulate the channel model consists of convolving the base band signal to be transmitted with the urban macro-cell or urban micro-cell channel model tap delay line generated using a MATLAB program (see Figure 7).

In order to verify the proper functioning of this method, a channel sounding based on a sliding correlation [19] is performed, with a sampling frequency $F_{e}$ of $64 \mathrm{MHz}$ at carrier frequency $f_{0}$ of $2.35 \mathrm{GHz}$. The sampling frequency is chosen initially higher, to obtain a good time resolution and verify that the channel is well emulated.

Figures $8(\mathrm{a})$ and $8(\mathrm{~b})$ present the power delay profile curves measured in RC urban micro- and urban macro-cell channel models, respectively.

The different results presented in this section highlight the possibility of controlling delay spread for each cluster, and emulating 3GPP urban micro- and macro-cell channel models. This is obtained by combining a digital preprocessing and a RC to manage the $\tau_{\text {rms }}$ value.

\section{LTE Active Measurement in Reverberation Chamber}

In order to realize these measurements, two boards representing a compact terminal are used: one reference board with one triband antenna, and diversity board with two triband antennas (see Figure 9). These antennas have been studied previously in $[20,21]$. The obtained characteristics are listed in Table 2.

4.1. SISO Measurement. By using the LTE system implemented in Section 2, the SISO configuration measurement will be performed. In order to simplify the analysis, the stirrers of the RC used for this study were rotated stepwisely for 1600 positions, so the Doppler spread of the channel is negligible. 


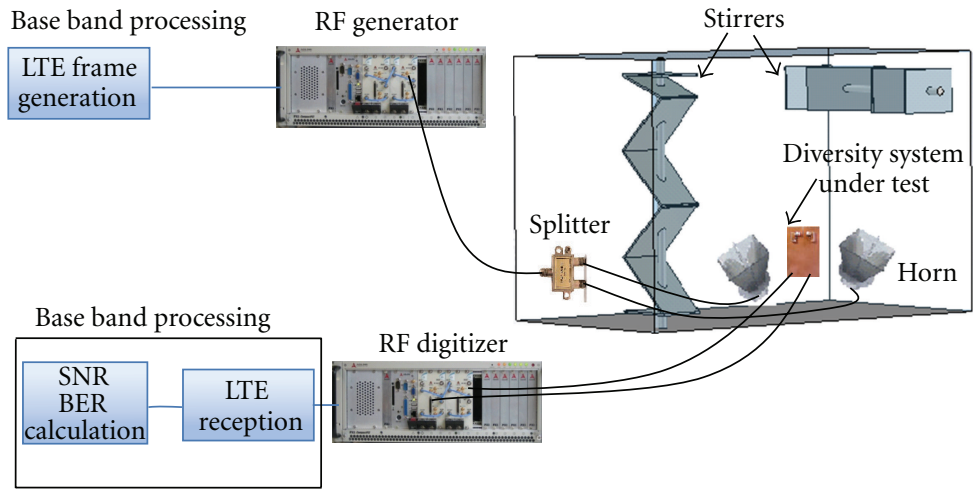

FIGURE 4: Reverberation chamber overview.

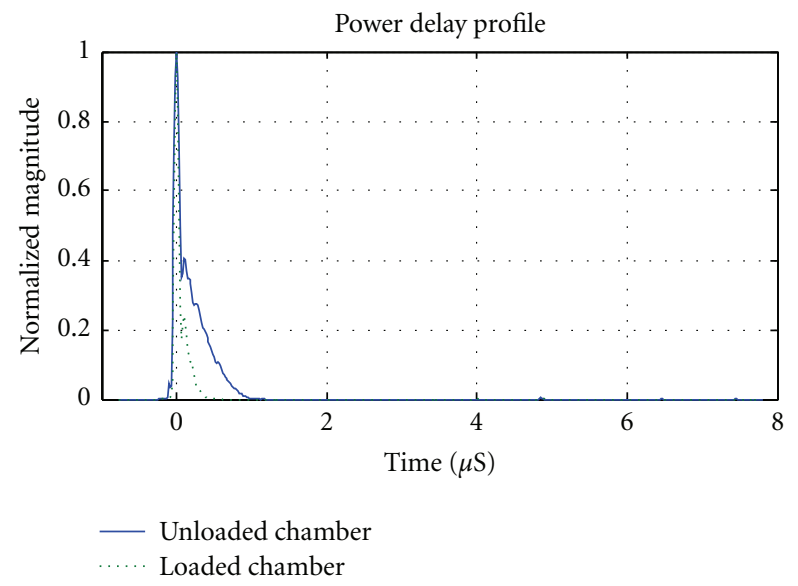

FIGURE 5: Measured PDPs for unloaded and loaded RC.

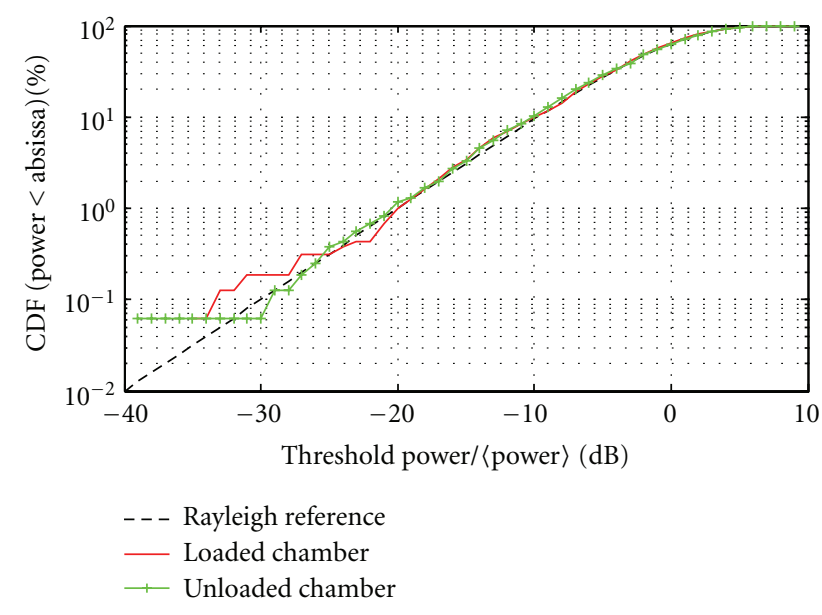

Figure 6: Cumulative distribution function measured in the loaded and unloaded RC. results:

The CDF curves plotted in Figure 10 present two types of

(i) cumulative distribution calculated from the measured power at one frequency in the signal bandwidth,

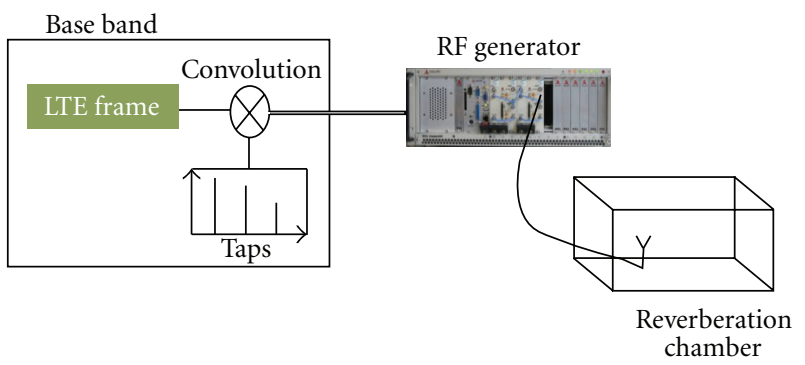

FIgURE 7: Channel emulation overview.

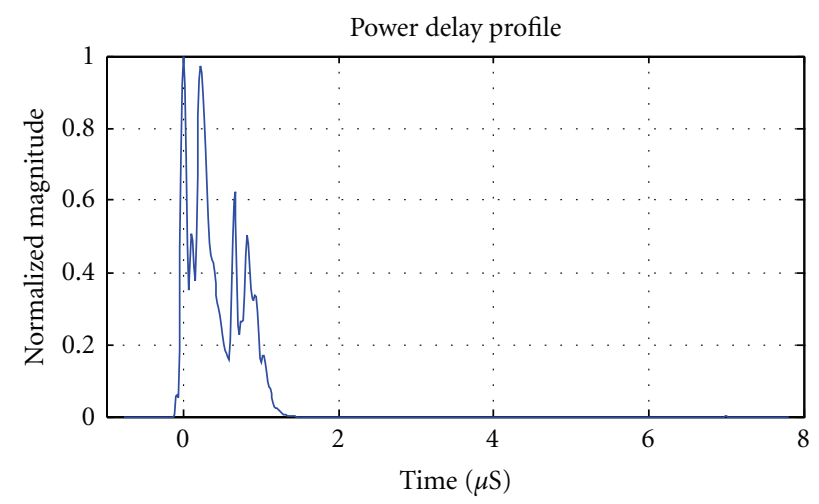

(a)

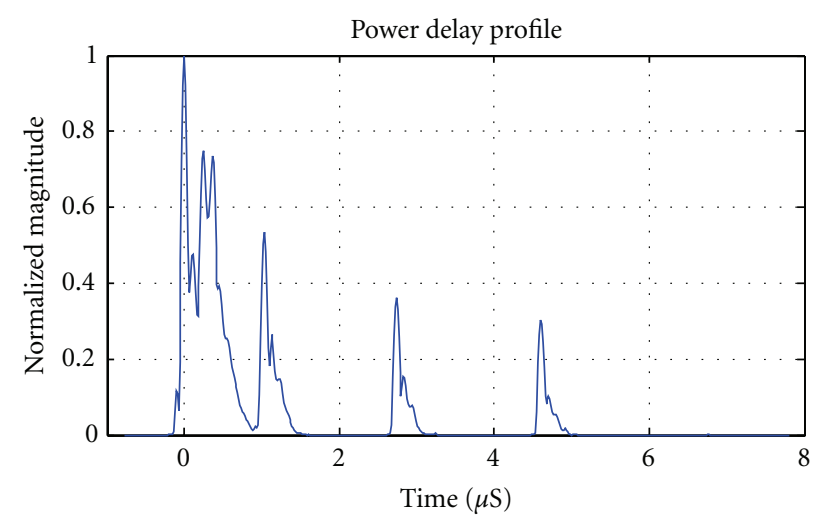

(b)

FIGURE 8: Power delay profiles measured in RC for urban micro-cell (a) and urban macro-cell (b) channel models. 


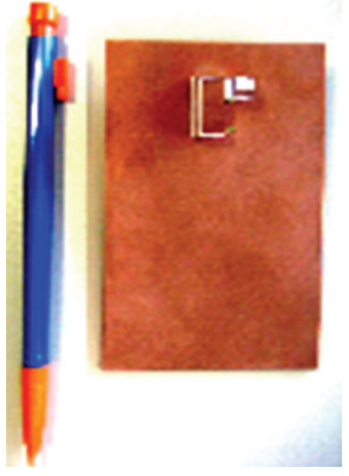

(a)

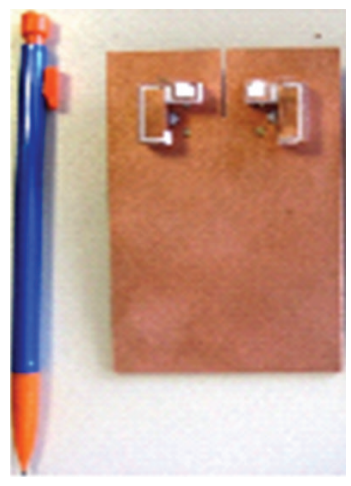

(b)
Figure 9: Reference antenna (a) and diversity antennas (b) [20, 21].

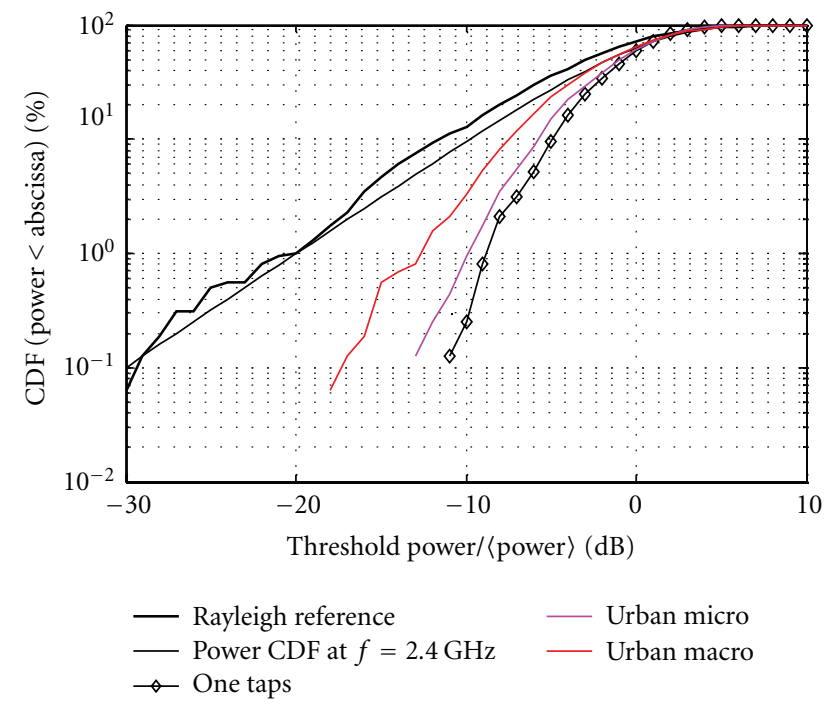

FIGURE 10: SISO cumulative distribution function measured in an RC for one cluster, urban micro-, and macrocell channel model cases.

(ii) the CDFs from the averaged power of received signal, demodulated after equalization.

For this comparison, each measured power of these results is normalized to its own average power.

As it is known OFDM signal gives better performance than using mono-carrier signal. It is an efficient way to deal against intersymbol interference, because such interference affects only a small percentage of the subcarriers. These interferences cause a frequency selectivity that can be well observed in Figure 10. Indeed it can be clearly seen that the cumulative power distributions are influenced by the delay spread of the channel model. The performance in urban micro- and urban macrocell channel models is worse compared to the monocluster case. Nevertheless, the received powers of the LTE signals are higher than those in the monocarrier case (which fit with the Rayleigh reference distribution).

These results can be confirmed by the signal-to-Noise ratio measurement, presented below. The estimation of the
TABLE 3: Measured mean SNR value for SISO configuration for a 64-QAM modulation scheme.

\begin{tabular}{lccc}
\hline & Monocluster & $\begin{array}{c}\text { Urban } \\
\text { microcell }\end{array}$ & $\begin{array}{c}\text { Urban } \\
\text { macrocell }\end{array}$ \\
\hline Mean SNR $(\mathrm{dB})$ & 25.7 & 7.9 & 6.4 \\
\hline
\end{tabular}

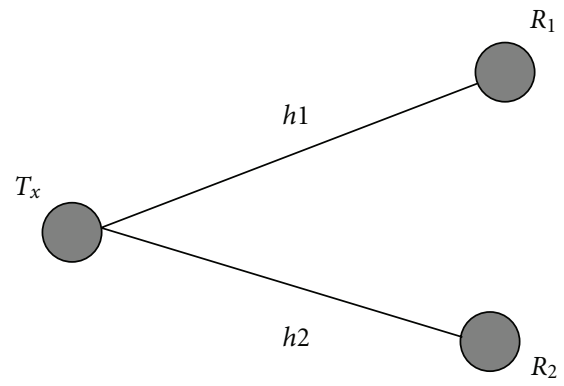

Figure 11: $1 \times 2$ Multiple antenna configuration.

measured mean SNR presented in Table 3 is done through the Error Vector Magnitude (EVM) [22]:

$$
\mathrm{EVM}=\left[\frac{(1 / N) \sum_{i=1}^{N}\left|S_{\text {ideal }, i}-S_{\text {meas }, i}\right|^{2}}{(1 / N) \sum_{i=1}^{N}\left|S_{\text {ideal }, i}\right|^{2}}\right]^{1 / 2}
$$

where $N$ is the number of symbols, $S_{\text {ideal }, i}$ denotes the ideal constellation point for the $i$ th symbol, and $S_{\text {meas, } i}$ is the measured $i$ th symbol.

The SNR is the inverse of the EVM taken in $\mathrm{dB}$, which is given by

$$
\mathrm{SNR}=20 \times \log _{10}\left(\frac{1}{\mathrm{EVM}}\right)
$$

The SISO measurement method allows assessing the capacity of a complete LTE transmission chain. This method makes it possible to know the performance as function of the receiver antenna, or base band processing (equalization, synchronization, etc.).

4.2. SIMO Measurement. In this section, the tests of the diversity systems performance are under interest. Diversity combining techniques such as Maximum Ratio Combining (MRC) have been implemented.

Multiple antennas are expressed as a $T \times R$ combination, where $T$ is the number of transmitting antennas, and $R$ is the number of the receiving antennas.

The example shown in Figure 11 presents a total of 2 links between transmit and receive antenna elements. These links are indicated by $h 1$ and $h 2$. These expressions are associated jointly to form an $H$ matrix (obtained by channel sounding measurements):

$$
H_{\text {meas }}=\left[\begin{array}{l}
h 1 \\
h 2
\end{array}\right]
$$




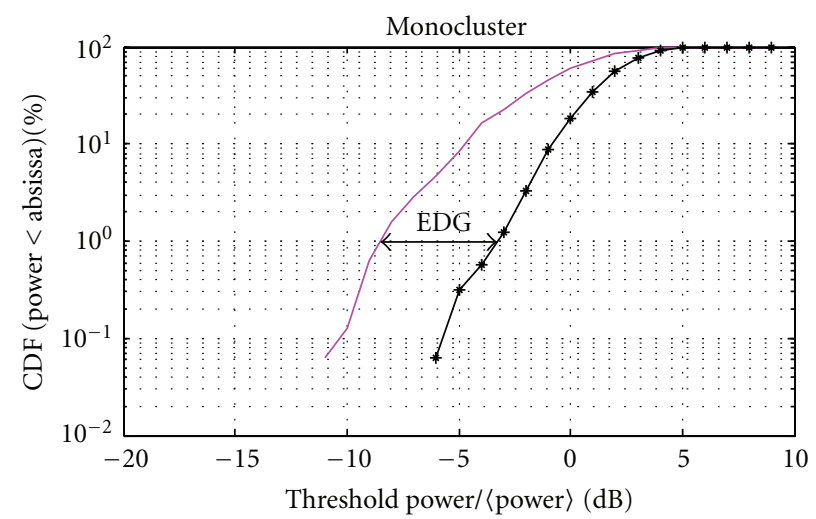

(a)

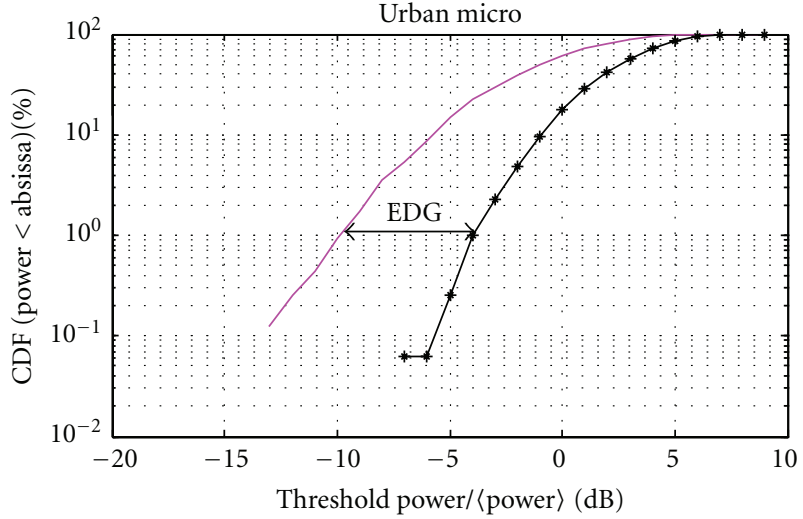

(b)

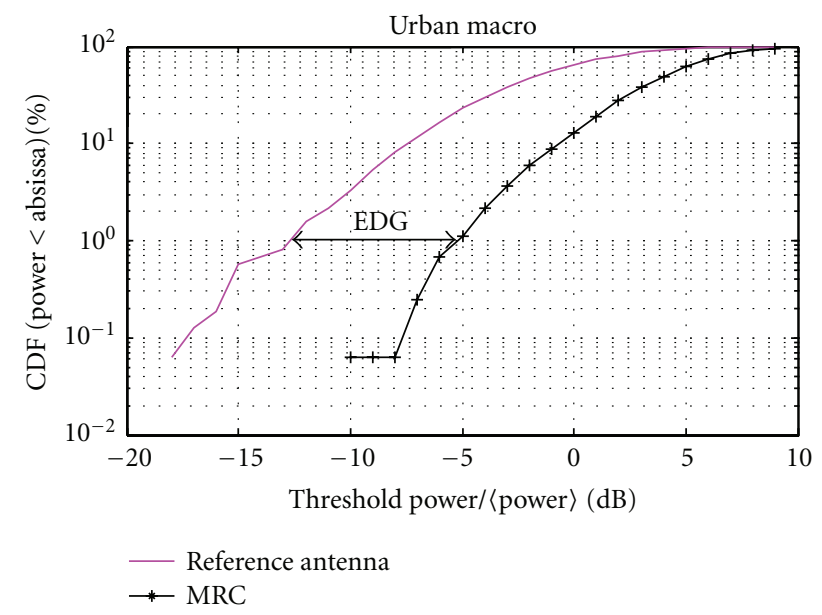

(c)

FIGURE 12: SIMO cumulative distribution function measured in an RC for one cluster (a), urban microcell (b), and urban macrocell (c) channel model cases.

The channel impulse responses taking in consideration multipath propagation phenomenon are presented by $h 1$ and $h 2$. The envelope correlation coefficient is as follows:

$$
\begin{gathered}
\rho=\frac{R(1,2)}{\sqrt{R(1,1) R(2,2)}}, \\
R=E\left\{\left(H_{\text {meas }}-E\left[H_{\text {meas }}\right]\right)\left(H_{\text {meas }}-E\left[H_{\text {meas }}\right]\right)^{H}\right\},
\end{gathered}
$$

where $R$ is the covariance matrix.

The calculation of the fading correlation coefficient for the different channel models generated in the $\mathrm{RC}$ is presented in Table 4.

It can be observed that for each channel model, the fading correlation remains low, which will lead to optimal diversity results.

Regarding the effective diversity gain (EDG), it is typically calculated for a particular frequency (passive measurements). EDG is the figure-of-merit (FOM) typically used to evaluate the efficiency of the diversity antenna system [14]. In our case, the evaluation of the EDG is made through the mean power of the received LTE signal in baseband.

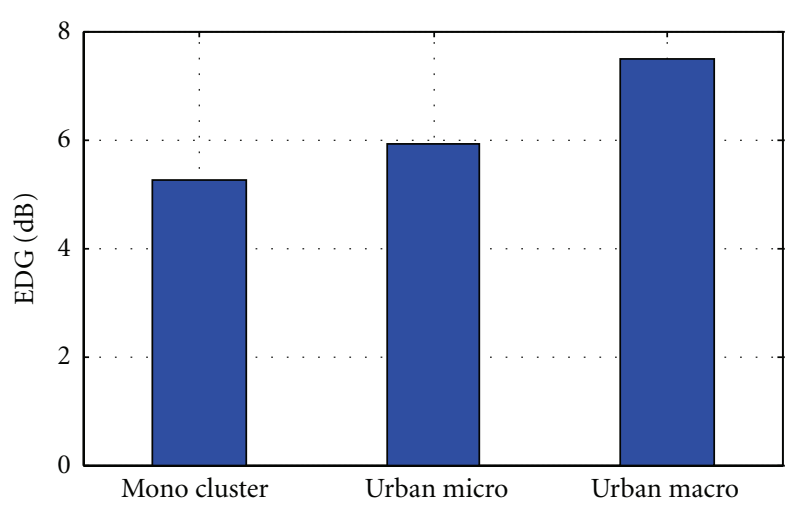

FIGURE 13: EDG for MRC technique at (1\%) for one cluster and urban micro- and macrocell channel model cases.

The CDFs of the received power (for the diversity and reference systems) are depicted in Figure 12. These results are normalized by the mean power received by the reference system in order to calculate the EDG at $1 \%$ probability (Figure 13). 
TABLE 4: Correlation coefficient.

\begin{tabular}{lccc}
\hline & \multicolumn{2}{c}{ Scenario } & \\
& One cluster & Urban micro & Urban macro \\
\hline $\begin{array}{l}\text { Fading correlation } \\
\text { coefficient }\end{array}$ & 0.15 & 0.21 & 0.32 \\
\hline
\end{tabular}

In conclusion, as we can observe in Figure 13 that the more significant improvement in terms of diversity gain is achieved for the macrourban channel case $(7.5 \mathrm{~dB})$. The Diversity Gain obtained for the monocluster case is less important, and it reaches $5.2 \mathrm{~dB}$. This limitation is due to the fact that the SNR of the SISO system in this case was already high when compared to the SNR in multipleclusters channels.

\section{Conclusions}

In this paper, we achieve to emulate the 3GPP channel models in a small reverberation cell. The performance of an LTE SISO/SIMO-OFDM system has been studied through active measurements for different channel models with Rayleigh fading distribution. The work developed in this paper permits to evaluate capacity of a wireless system in controlled environments and gives the opportunity to test different transmission chain parameters (base band processing, modulation scheme, antennas, etc.) in the same environment and compare their performance. This approach could be of particular interest for OTA characterization of multiantenna terminals in reverberation chambers, because it helps to understand the influence of the channel characteristics under which measurements have been performed.

Future works will consist in making experiments with specific MIMO schemes, based on LTE specifications. Regarding the control of the delay spread, an analytical method will be developed to estimate it in function of the amount of absorbing material inside the RC. This method will lead to avoid channel sounding measurements, currently needed to achieve the desired delay spread.

\section{References}

[1] J. F. Valenzuela-Valdès, A. M. Martinez-Gonzalez, and D. A. Sanchez-Hernandez, "Diversity gain and MIMO capacity for non isotropic environments using a reverberation chamber," Antennas and Wireless Propagation Letters, IEEE, vol. 8, pp. $112-115,2009$.

[2] D. A. Hill, M. T. Ma, A. R. Ondrejka, B. F. Riddle, M. L. Crawford, and R. T. Johnk, "Aperture excitation of electrically large, lossy cavities," IEEE Transactions on Electromagnetic Compatibility, vol. 36, no. 3, pp. 169-178, 1994.

[3] J. D. Sanchez-Heredia, J. F. Valenzuela-Valdès, A. M. MartinezGonzalez, and D. A. Sanchez-Hernandez, "Emulation of MIMO rician fading environments with mode-stirred reverberation chambers," IEEE Transactions on Antennas and Propagation, vol. 59, no. 2, pp. 654-660, 2011.

[4] X. Chen, P. S. Kildal, C. Orlenius, and J. Carlsson, "Channel sounding of loaded reverberation chamber for over-the-air testing of wireless devices: coherence bandwidth versus average mode bandwidth and delay spread," IEEE Antennas and Wireless Propagation Letters, vol. 8, Article ID 5072260, pp. 678-681, 2009.

[5] 3GPP TS 36.101, Evolved Universal Terrestrial Radio Access (E.UTRA); User Equipment (UE) radio transmission and reception (Release 9), version9.3.0, Mars 2010.

[6] 3GPP TR 37.976, Measurement of radiated performance for MIMO and multi-antenna reception for HSPA and LTE terminals (Release 10), version 1.1.0, May 2010.

[7] A. Diallo, C. Luxey, P. L. Thuc, R. Straj, and G. Kossiavas, "Diversity performance of multiantenna systems for UMTS cellular phones in different propagation environments," International Journal of Antennas and Propagation, vol. 2008, Article ID 836050, 10 pages, 2008.

[8] A. Pascual-Iserte, L. M. Ventura, and X. Nieto, "Residual carrier frequency offset estimation and correction in OFDM MIMO systems," in Proceedings of the 18th Annual IEEE International Symposium on Personal, Indoor and Mobile Radio Communications (PIMRC'07), pp. 1-5, Athens, Greece, September 2007.

[9] S. Blanch, J. Romeu, and I. Corbella, "Exact representation of antenna system diversity performance from input parameter description," Electronics Letters, vol. 39, no. 9, pp. 705-707, 2003.

[10] LTE forum technology, http://www.3gpp.org/.

[11] M. B. Knudsen and G. F. Pedersen, "Spherical outdoor to indoor power spectrum model at the mobile terminal," IEEE Journal on Selected Areas in Communications, vol. 20, no. 6, pp. 1156-1169, 2002.

[12] T. Bolin, A. Derneryd, G. Kristensson, V. Plicanic, and Z. Ying, "Two-antenna receive diversity performance in indoor environment," Electronics Letters, vol. 41, no. 22, pp. 1205-1206, 2005.

[13] R. G. Vaughan and J. B. Andersen, "Antenna diversity in mobile communication," IEEE Transactions on Vehicular Technology, vol. 36, no. 4, pp. 149-172, 1987.

[14] P. S. Kildal, K. Rosengren, J. Byun, and J. Lee, "Definition of effective diversity gain and how to measure it in a reverberation chamber," Microwave and Optical Technology Letters, vol. 34, no. 1, pp. 56-59, 2002.

[15] T. Taga, "Analysis for mean effective gain of mobile antennas in land mobile radio environments," IEEE Transactions on Vehicular Technology, vol. 39, no. 2, pp. 117-131, 1990.

[16] A. Belhouji, C. Decroze, D. Carsenat, M. Mouhamadou, and T. Monediere, "Active measurements of a MIMO WiMAXOFDM based system in reverberation chambers," Microwave and Optical Technology Letters, vol. 52, no. 10, pp. 2347-2352, 2010.

[17] ETS-Lindgren's, "SMART 1000 Mini-Reverb Cell," http:// www.ets-lindgren.com/pdf/smart1000.pdf.

[18] http://www.ecanechoicchambers.com/.

[19] J. C. Lin and C. Y. Lin, "Noncoherent sequential PN code acquisition using sliding correlation in DS/SS," in Proceedings of the IEEE International Conference on Communications (ICC'00), vol. 1, pp. 341-345, New Orleans, La , USA, June 2000.

[20] M. Mouhamadou, M. Koubeissi, C. Tounou et al., "Multiband diversity antenna performances evaluation for multistandard compact wireless terminal," in Proceedings of the 3 rd European Conference on Antennas and Propagation (EuCAP '09), Berlin, Germany, March 2009. 
[21] M. Mouhamadou, M. Koubeissi, C. Tounou et al., "Multiband diversity antenna performances evaluation for multistandard compact wireless terminal," in Proceedings of the $3 \mathrm{rd}$ European Conference on Antennas and Propagation (EuCAP '09), Berlin, Germany, March 2009.

[22] R. A. Shafi, S. Rahman, and A. H. M. Razibul, "On the extended relationships among EVM, BER and SNR as performance metrics," in Proceedings of the International Conference on Electrical and computer Engineering (ICECE '06), Dhaka, Bangladesh, December 2006. 

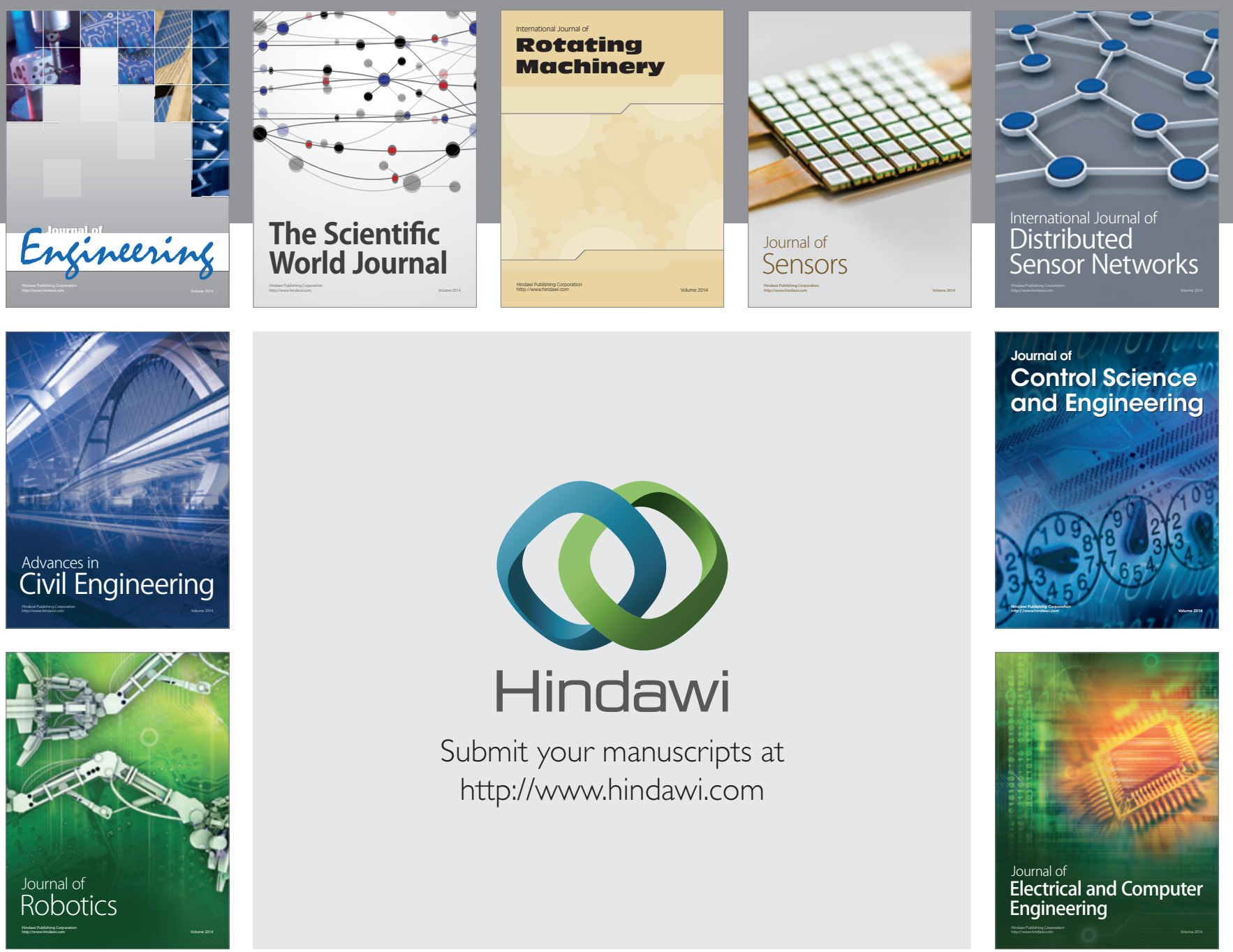

Submit your manuscripts at

http://www.hindawi.com
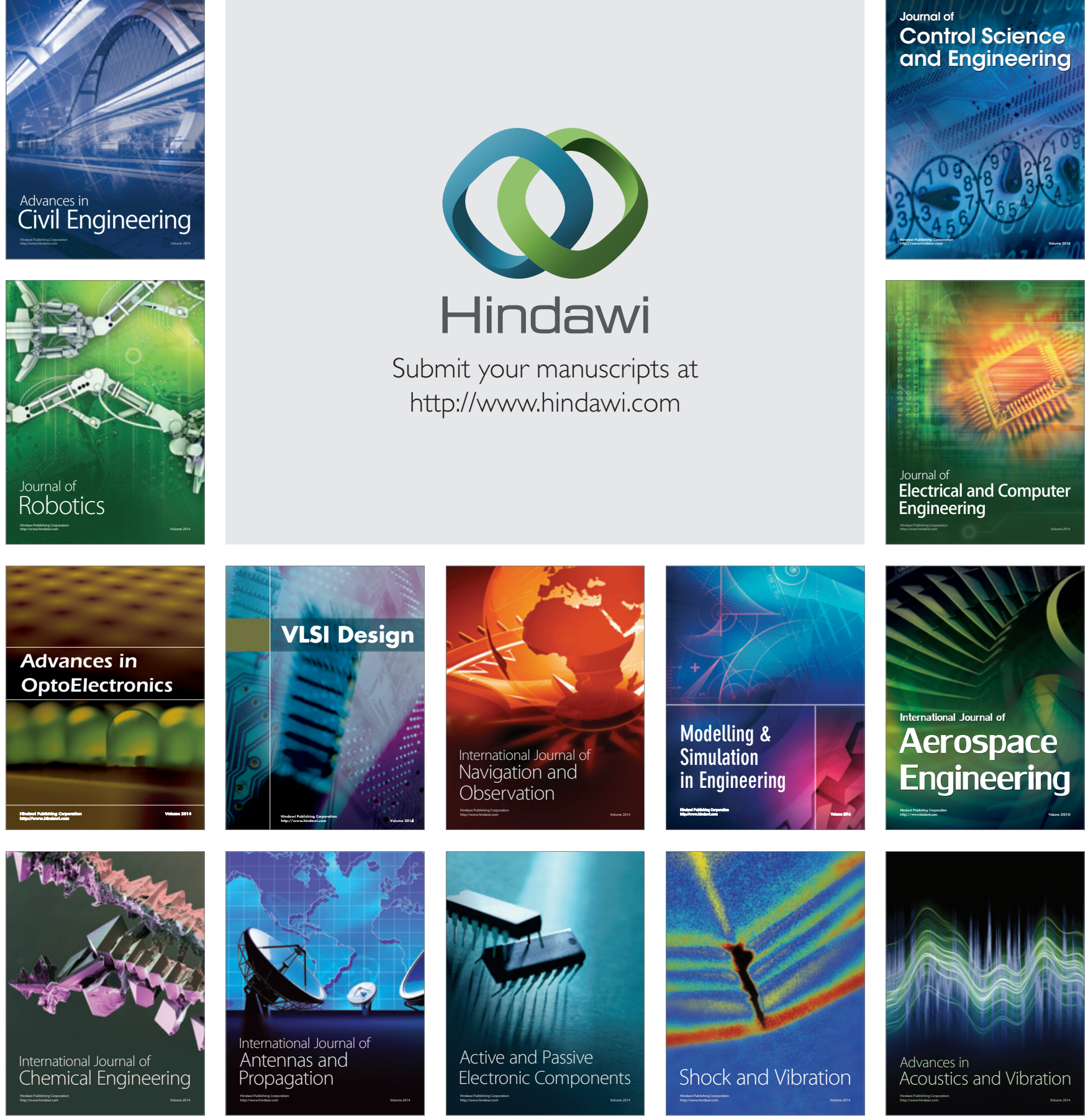